\title{
Local immunodiagnosis of pulmonary tuberculosis by enzyme-linked immunospot
}

\author{
C. Jafari*, M. Ernst” ${ }^{\#}$ A. Strassburg*, U. Greinert*, B. Kalsdorf*, \\ D. Kirsten ${ }^{\star}$ and C. Lange*
}

ABSTRACT: Lymphocytes are crucial in the immune defence against Mycobacterium tuberculosis (MTB) infection. The aim of the present study was to ascertain whether or not MTB-specific lymphocytes are selectively compartmentalised in the lungs of patients with minimal active pulmonary tuberculosis (PTB).

Patients with smear-negative MTB-culture-confirmed PTB were prospectively recruited. Differential cell counts, immunophenotyping with monoclonal antibodies directed against the cell surface markers CD4, CD8, CD4CD45RA, CD4CD45R0, CD38, human leukocyte antigen DR, CD19, CD3, CD57 and CD16 and MTB-specific enzyme-linked immunospot assays of peripheral blood mononuclear cells and bronchoalveolar lavage (BAL) mononuclear cells with 6-kDa early secretory antigenic target and culture filtrate protein 10 were performed.

Among 12 patients with culture-confirmed smear-negative PTB, no differences were found in the distribution of total CD4 or CD8 T-cells in peripheral blood or BAL fluid (BALF). Activated human leukocyte antigen-DR-positive cells, as well as memory CD4CD45R0-positive T-cells, were expanded among cells of the BALF. Compared with a group of control patients with alternative pulmonary pathologies, there was no significant difference in lymphocyte subpopulations. However, 6-kDa early secretory antigenic target- and culture filtrate protein 10-specific lymphocytes were more concentrated, with a median BALF:peripheral blood ratio of 9.9 and 8.9, respectively, in patients with PTB.

Mycobacterium tuberculosis-specific T-cells are highly selectively compartmentalised at the site of infection in active pulmonary tuberculosis.

KEYWORDS: Bronchoalveolar lavage, culture filtrate protein 10, enzyme-linked immunospot, lymphocytes, 6-kDa early secretory antigenic target, tuberculosis

$\mathbf{T}$ -lymphocytes play an important role in the adaptive immune defence against Mycobacterium tuberculosis (MTB) by direct interaction with alveolar macrophages [1, 2].

In healthy adult humans, the total number of Tlymphocytes is estimated to be $3.0 \times 10^{11}$ [3]. Approximately $2 \%$ of T-lymphocytes are found in the peripheral blood, whereas nearly 10\% reside in the lungs [3]. In active tuberculosis (TB), memory T-cells clonally expand upon antigen encounter and are recruited to the site of the infection [4-10]. These MTB-specific memory Tcells produce interferon (IFN)- $\gamma$ [11] and other cytokines of the type-1 T-helper cell-type immune response [12]. Prompt production of IFN- $\gamma$ following specific antigen presentation is predominantly provided by the population of effector T-cells [11], which typically have a CD4CD45R0-positive, CD27-negative and CC chemokine receptor 7-negative phenotype and are end-differentiated for a rapid, antigen-specific cytotoxic immune response [11, 13].

Since the late 1990s, major advances have been made in the immunodiagnosis of MTB infection with the development of T-cell IFN- $\gamma$ release assays. Enumeration of 6-kDa early secretory antigenic target (ESAT)-6 and culture filtrate protein (CFP)-10-specific T-lymphocytes from peripheral blood by enzyme-linked immunospot (ELISPOT) assay or ELISA has been shown to be more sensitive and specific for the immunodiagnosis of active and latent TB infection (LTBI) than the tuberculin skin test (TST) [14-18]. However, since effector T-cells only occur at low frequency in peripheral blood, it is not surprising that a clear distinction between active TB, LTBI and previously treated TB has not been possible when Tcell IFN- $\gamma$ release assays have been performed with cells from the peripheral blood alone [19, 20]. In contrast, counting antigen-specific cells from
AFFILIATIONS

Divisions of ${ }^{*}$ Clinical Infectious

Diseases, and

\# Immune Cell Analytics, Borstel

Research Center, Borstel, and

" Center for Pulmonary Medicine and Thoracic Surgery, Großhansdorf Hospital, Hamburg, Germany.

CORRESPONDENCE

C. Lange

Division of Clinical Infectious Diseases

Research Center Borstel

Parkallee 35

23845 Borstel

Germany

Fax: 494537188313

E-mail: clange@fz-borstel.de

Received:

July 302007

Accepted after revision:

October 172007

STATEMENT OF INTEREST

None declared.
European Respiratory Journal Print ISSN 0903-1936 Online ISSN 1399-3003 
the site of the infection has been shown to be a promising method for distinguishing active TB infection from LTBI [21-24].

In order to gain a better understanding about the magnitude of recruitment of lymphocytes from the blood into the human lung during active pulmonary TB (PTB), immunophenotypes and numbers of MTB-specific T-lymphocytes were analysed in prospectively recruited patients with a suspected diagnosis of PTB and negative acid-fast bacillus sputum microscopy results.

\section{METHODS}

\section{Subjects}

Patients with three negative acid-fast bacillus smear results, pulmonary infiltrates on chest radiography or thoracic computed tomography and suspected PTB were enrolled. Peripheral blood mononuclear cells (PBMCs) were obtained by venous blood draw. Bronchoalveolar lavage mononuclear cells (BALMCs) were obtained by bronchoscopy [25]. Only patients with culture-confirmed smear-negative active PTB were assigned to the TB group; all patients with presumptive but smear- and culture-negative TB were excluded from the study. Patients with negative MTB cultures and an alternative diagnosis were assigned to the non-TB group. Healthy controls were not included in the study. The study was approved by the ethics committee of the University of Lübeck Medical School (Lübeck, Germany), and all patients gave written informed consent.

\section{Immunophenotyping}

Lymphocyte subsets were enumerated among PBMCs obtained from freshly drawn blood and BALMCs using directly labelled murine monoclonal antibodies directed against CD4, CD8, naive CD4CD45RA, memory CD4CD45R0, CD38, human leukocyte antigen DR, CD19, CD3, CD57 and CD16 (DakoCytomation, Hamburg, Germany) by two-colour flow cytometry (FACSCalibur; Becton-Dickinson, Heidelberg, Germany).

\section{Cell preparations and ELISPOT assays}

Cell preparations for ELISPOT assays of human IFN- $\gamma$ were performed as described previously [22]. The numbers of spot-forming cells (SFCs) per million lymphocytes were calculated from net numbers of SFCs among PBMCs and BALMCs by means of blood and BAL fluid (BALF) differential cell counts.

\section{Statistical analysis}

Statistical tests were performed using nonparametric testing for exploratory analyses, without adjustment for multiple testing, with nominal significance defined as having a p-value of $<0.05$. In order to avoid mathematical errors due to division by zero, a value of 0 was assigned a value of 0.1 when calculating ratios of ESAT-6- and CFP-10-specific cells among lymphocytes among PBMCs and BALMCs.

\section{RESULTS}

\section{Patient characteristics}

Forty patients with suspected PTB were prospectively enrolled. Twelve had the diagnosis of PTB confirmed by positive MTB culture from sputum or BALF (eight of these patients had been included in a previous report [22]). A group of 25 patients with negative MTB culture results were diagnosed with aetiologies other than PTB; nine of which had $<1 \%$ lymphocytes in their BALF or not enough BALF available for the fluorescence-activated cell sorter analysis and so also had to be excluded from the study. The diagnoses of the remaining 16 patients from the non-TB control group were sarcoidosis $(n=4)$, pneumonia $(n=4)$, former mycobacterial infection without reactivation $(n=4)$, cryptogenic organising pneumonia $(n=2)$, bronchogenic carcinoma $(n=1)$ and rheumatoid arthritis $(n=1)$. Seven non-TB patients gave positive ELISPOT results with peripheral blood, and were diagnosed with LTBI. Patients with PTB were younger (31.1 versus 50.7 yrs; $p<0.018$ ) and more often male (10 out of 12$)$ than patients with alternative diseases $(10$ out of 16 ; $p<0.021)$.

\section{ELISPOT assay results}

The median number of ESAT-6- and CFP-10-specific lymphocytes in patients with PTB was 64 (interquartile range (IQR) 35-167) and 149 (85-259) per million lymphocytes,

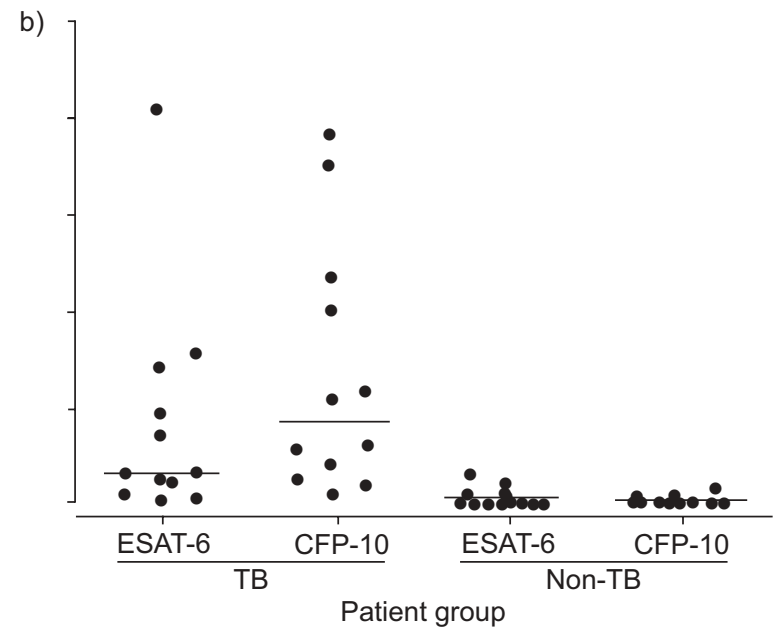

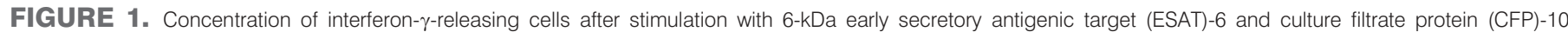

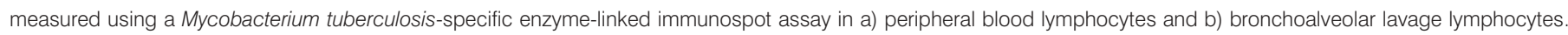
Horizontal bars represent medians. TB: tuberculosis; SFC: spot-forming cell. 


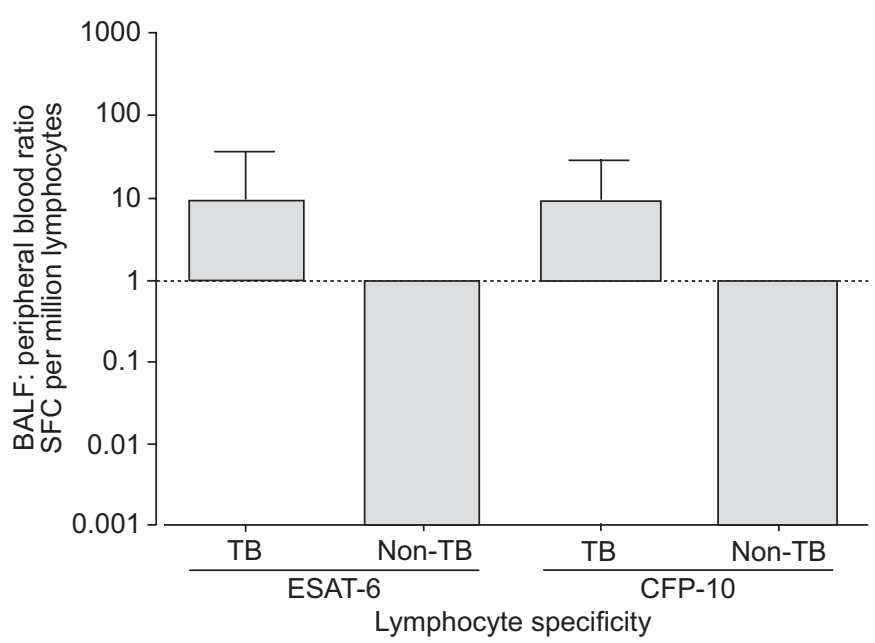

FIGURE 2. Ratio of 6-kDa early secretory antigenic target (ESAT)-6- and culture filtrate protein (CFP)-10-specific lymphocytes in bronchoalveolar lavage fluid (BALF) and peripheral blood (----: 1:1 ratio). Data are presented as median and interquartile range. The BALF: peripheral blood ratio is close to 0 for both antigens in non-tuberculosis (TB) patients. SFC: spot-forming cell.

respectively, in the peripheral blood, and $598(244-2,593)$ and 1,653 $(549-4,511)$ per million lymphocytes, respectively, in BALF. The median number of ESAT-6- and CFP-10-specific lymphocytes in patients in the non-TB group was $3(0-33)$ and $6(0-32)$ per million lymphocytes, respectively, in the peripheral blood, and $0(0-150)$ and $0(0-73)$ per million lymphocytes, respectively, in BALF (fig. 1).

\section{Compartmentalisation of ESAT-6- and CFP-10-specific lymphocytes in the lungs of patients with PTB}

In patients with $\mathrm{PTB}$, the median (IQR) BALF:peripheral blood ratio of ESAT-6- and CFP-10-specific SFCs per million lymphocytes was 9.9 (3-37) and 8.9 (6-32), respectively. In contrast, the median BALF:peripheral blood ratio of ESAT-6and CFP-10-specific SFCs per million lymphocytes in control patients was $0(0-13)$ and $0(0-7)$, respectively (fig. 2).

\section{Immunophenotype}

Blood and BALF immunophenotypes are shown in table 1. There was no significant difference in the immunophenotyping results of PBMCs or BALMCs between patients with PTB and controls (data not shown).

\section{DISCUSSION}

Since the late 1990s, an MTB-specific ELISA (QuantiFERONTB-Gold In-Tube; Cellestis, Carnegie, Australia) and ELISPOT assay (T-Spot.TB; Oxford Immunotec, Abingdon, UK) have been developed for the diagnosis of TB [26-28]. However, there has been surprisingly little discussion as to what these assays really measure. Although the ELISA quantifies IFN- $\gamma$ production following MTB-specific antigen contact in whole blood, it is possible to enumerate single MTB-specific cells using the ELISPOT assay. Since antigen-presenting monocytes and dendritic cells, which are not a source of IFN- $\gamma$ production, are assayed together with lymphocytes in the ELISPOT assay, ELISPOT assay results should be normalised to lymphocyte numbers in order to achieve more accurate results. Only a minority of the lymphocytes in the human body are found in peripheral blood [29], and the frequency with which MTBspecific T-cells occur in peripheral blood is very low $(\sim 0.002$ $0.4 \%$ with respect to ESAT-6- and CFP-10-specific T-cells) even in active TB [21, 22]. It is not surprising, therefore, that it has not been possible to discriminate between active TB and LTBI in routine clinical practice using these assays [27].

Following antigen encounter, naïve cytotoxic T-lymphocyte precursors are primed and acquire effector function. They undergo expansion and travel to sites of infection, where they mediate pathogen clearance by killing infected cells and

TABLE 1 Peripheral blood mononuclear cell (PBMC) and bronchoalveolar lavage mononuclear cell (BALMC) immunophenotypes in patients with culture-positive tuberculosis (TB) and other pulmonary diseases

\begin{tabular}{|c|c|c|c|c|c|c|}
\hline \multirow[t]{2}{*}{ Lymphocyte surface marker } & \multicolumn{3}{|c|}{ TB group } & \multicolumn{3}{|c|}{ Non-TB group } \\
\hline & PBMCs & BALMCs & p-value & PBMCs & BALMCs & $\mathrm{p}$-value \\
\hline CD4 & $38(26-49)$ & $52(20-59)$ & NS & $32(21-42)$ & $37(20-58)$ & NS \\
\hline CD8 & $39(36-51)$ & $35(31-67)$ & NS & $40(29-48)$ & $34(22-54)$ & NS \\
\hline CD38 & 55 (43-69) & $35(26-60)$ & 0.012 & $50(40-66)$ & $34(24-44)$ & 0.041 \\
\hline HLA-DR & $33(24-52)$ & $48(41-80)$ & 0.017 & $33(24-41)$ & $59(37-70)$ & 0.031 \\
\hline CD19 & $11(8-17)$ & $2(1-5)$ & 0.005 & $11(7-24)$ & $4(3-6)$ & 0.027 \\
\hline CD3 & $75(62-78)$ & 76 (75-84) & NS & 69 (47-74) & $75(59-85)$ & NS \\
\hline CD57 & $34(17-53)$ & $22(19-32)$ & NS & $33(20-50)$ & $32(23-44)$ & NS \\
\hline CD16 & $12(11-20)$ & $5(1-8)$ & 0.007 & $18(12-31)$ & $7(5-14)$ & 0.008 \\
\hline
\end{tabular}


secreting effector cytokines. Subsequently, most effector cytotoxic T-lymphocytes die [30]. It may, therefore, be possible to discriminate active TB from LTBI using immunodiagnosis, comparing the frequencies of antigen-specific cells from the site of infection and peripheral blood using MTB-specific ELISPOT analysis [21, 22]. Compartmentalisation of antimycobacterial immune responses at the site of infection during active TB has previously been described [8, 12, 21, 31-38]. In healthy household contacts of patients with TB [33], and in patients with active PTB, antigen-specific lymphocytes are concentrated in the lungs, and, on challenge, produce type- 1 helper T-cell cytokine host responses [34]. However, to date, the magnitude of antigen-specific lymphocyte concentration has not been quantified in PTB.

In the present study, MTB-specific T-lymphocytes were found to be concentrated by a factor of approximately log 1 among lymphocytes derived by BAL from patients with active smearnegative PTB compared with those from the peripheral blood. This concentration of MTB-specific T-cells among lymphocytes in BALF compared with peripheral blood is of the same order of magnitude as the concentration of MTB-specific T-cells among lymphocytes in pleural effusion compared with peripheral blood in TB pleurisy [21]. It is of note that, in agreement with previous findings [6], no other differences in immunophenotype among PBMCs or BALMCs from patients with active PTB and controls could be found in the present study.

When the immunophenotype of PBMCs and BALMCs were directly compared, there were higher frequencies of CD4CD45R0 memory T-cells and human leukocyte antigen DR-positive activated T-cells and a lower frequency of CD4CD45RA-naive T-cells, CD19 B-cells and CD16 natural killer cells among BALMCs than among PBMCs, consistent with earlier reports $[6,39,40]$. However, these differences were not restricted to patients with $\mathrm{PTB}$; the same differences between PBMCs and BALMCs were also observed in patients with other pulmonary pathologies. In active PTB, antigenspecific T-cells are, therefore, expanded among other lymphocytes in the lungs, and it is possible that most of the lymphocytes found at the site of infection in PTB are, indeed, not MTB-specific. The limitations of the present study need to be addressed. An influence of age or sex on MTB-specific immune responses cannot be excluded. Only patients with limited smear-negative TB were included in the present study. The findings regarding immunophenotypes may have been different if patients with more advanced disease or with immunosuppression had been included. It also remains unclear as to whether the concentration of MTB-specific Tcells at the site of infection is due to active recruitment of circulating cells or local proliferation and expansion of a few specific precursors or a combination of both [32].

In conclusion, antigen-specific T-lymphocytes are specifically concentrated at the site of infection in active $\mathrm{TB}$, and discrimination of active PTB from LTBI may be possible by comparing the frequencies of antigen-specific T-lymphocytes in peripheral blood and in BALF lymphocytes by ELISPOT assay. As high numbers of antigen-presenting cells may be present among mononuclear cells from extrasanguineous compartments, MTB-specific ELISPOT assay results should be normalised to the numbers of lymphocytes in the assay.

Future studies should evaluate whether local immunodiagnosis of tuberculosis by enzyme-linked immunospot assay is also appropriate for extrapulmonary manifestations of tuberculosis, such as meningitis, peritonitis or pericarditis.

\section{REFERENCES}

1 Chan J, Flynn J. The immunological aspects of latency in tuberculosis. Clin Immunol 2004; 110: 2-12.

2 Kaufmann SH. How can immunology contribute to the control of tuberculosis? Nat Rev Immunol 2001; 1: 20-30.

3 Rannie GH, Donald KJ. Estimation of the migration of thoracic duct lymphocytes to non-lymphoid tissues. A comparison of the distribution of radioactivity at intervals following i.v. transfusion of cells labelled with ${ }^{3} \mathrm{H},{ }^{14} \mathrm{C}$, ${ }^{75} \mathrm{Se},{ }^{99 \mathrm{~m}} \mathrm{Tc},{ }^{125} \mathrm{I}$ and ${ }^{51} \mathrm{Cr}$ in the rat. Cell Tissue Kinet 1977 ; 10: 523-541.

4 Barnes PF, Mistry SD, Cooper CL, Pirmez C, Rea TH, Modlin RL. Compartmentalization of a CD4+ T lymphocyte subpopulation in tuberculous pleuritis. J Immunol 1989; 142: 1114-1119.

5 Dhand R, De A, Ganguly NK, et al. Factors influencing the cellular response in bronchoalveolar lavage and peripheral blood of patients with pulmonary tuberculosis. Tubercle 1988; 69: 161-173.

6 Hoheisel GB, Tabak L, Teschler H, Erkan F, Kroegel C, Costabel U. Bronchoalveolar lavage cytology and immunocytology in pulmonary tuberculosis. Am J Respir Crit Care Med 1994; 149: 460-463.

7 Mazzarella G, Bianco A, Perna F, et al. T lymphocyte phenotypic profile in lung segments affected by cavitary and non-cavitary tuberculosis. Clin Exp Immunol 2003; 132: 283-288.

8 Silver RF, Zukowski L, Kotake S, et al. Recruitment of antigen-specific Th1-like responses to the human lung following bronchoscopic segmental challenge with purified protein derivative of Mycobacterium tuberculosis. Am J Respir Cell Mol Biol 2003; 29: 117-123.

9 Tsao TC, Tsao KC, Lin MC, et al. Increased absolute number but not proportion of $\gamma / \delta$ T-lymphocytes in the bronchoalveolar lavage fluid of patients with active pulmonary tuberculosis. Tuber Lung Dis 1999; 79: 215-220.

10 Uh ST, Ki SY, Lim GI, et al. The T cell receptor subsets of lymphocytes in bronchoalveolar lavage in patients with active pulmonary tuberculosis. Respir Med 1998; 92: 408-414.

11 Lalvani A, Brookes R, Hambleton S, Britton WJ, Hill AVS, McMichael AJ. Rapid effector function in CD8+ memory T cells. J Exp Med 1997; 186: 859-865.

12 Walrath J, Zukowski L, Krywiak A, Silver RF. Resident Th1-like effector memory cells in pulmonary recall responses to Mycobacterium tuberculosis. Am J Respir Cell Mol Biol 2005; 33: 48-55.

13 Goletti D, Butera O, Bizzoni F, Casetti R, Girardi E, Poccia F. Region of difference 1 antigen-specific CD4+ memory $\mathrm{T}$ cells correlate with a favorable outcome of tuberculosis. J Infect Dis 2006; 194: 984-992. 
14 Brock I, Weldingh K, Lillebaek T, Follmann F, Andersen P. Comparison of tuberculin skin test and new specific blood test in tuberculosis contacts. Am J Respir Crit Care Med 2004; 170: 65-69.

15 Ferrara G, Losi M, D'Amico R, et al. Use in routine clinical practice of two commercial blood tests for diagnosis of infection with Mycobacterium tuberculosis: a prospective study. Lancet 2006; 367: 1328-1334.

16 Kang YA, Lee HW, Yoon HI, et al. Discrepancy between the tuberculin skin test and the whole-blood interferon $\gamma$ assay for the diagnosis of latent tuberculosis infection in an intermediate tuberculosis-burden country. JAMA 2005; 293: 2756-2761.

17 Lalvani A, Pathan AA, McShane H, et al. Rapid detection of Mycobacterium tuberculosis infection by enumeration of antigen-specific T cells. Am J Respir Crit Care Med 2001; 163: 824-828.

18 Ravn P, Munk ME, Andersen $\mathrm{AB}$, et al. Prospective evaluation of a whole-blood test using Mycobacterium tuberculosis-specific antigens ESAT-6 and CFP-10 for diagnosis of active tuberculosis. Clin Diagn Lab Immunol 2005; 12 : 491-496.

19 Menzies D, Pai M, Comstock G. Meta-analysis: new tests for the diagnosis of latent tuberculosis infection: areas of uncertainty and recommendations for research. Ann Intern Med 2007; 146: 340-354.

20 Pai M, Menzies D. Interferon- $\gamma$ release assays: what is their role in the diagnosis of active tuberculosis? Clin Infect Dis 2007; 44: 74-77.

21 Wilkinson KA, Wilkinson RJ, Pathan A, et al. Ex vivo characterization of early secretory antigenic target 6specific $\mathrm{T}$ cells at sites of active disease in pleural tuberculosis. Clin Infect Dis 2005; 40: 184-187.

22 Jafari C, Ernst M, Kalsdorf B, et al. Rapid diagnosis of smear-negative tuberculosis by bronchoalveolar lavage enzyme-linked immunospot. Am J Respir Crit Care Med 2006; 174: 1048-1054.

23 Lange C, Hellmich B, Ernst M, Ehlers S. Rapid immunodiagnosis of tuberculosis in a woman receiving anti-TNF therapy. Nat Clin Pract Rheumatol 2007; 3: 528-534.

24 Strassburg A, Jafari C, Ernst M, Lange C. Rapid diagnosis of pulmonary tuberculosis by BAL-ELISPOT in an immunocomromised host. Eur Respir J 2008; (in Press).

25 Haussinger K, Ballin A, Becker HD, et al. Empfehlungen zur Sicherung der Qualität in der Bronchoskopie. [Recommendations for quality standards in bronchoscopy.] Pneumologie 2004; 58: 344-356.

26 Lalvani A. Diagnosing tuberculosis infection in the 21st century: new tools to tackle an old enemy. Chest 2007; 131: 1898-1906.
27 Pai M, Riley LW, Colford JM Jr. Interferon- $\gamma$ assays in the immunodiagnosis of tuberculosis: a systematic review. Lancet Infect Dis 2004; 4: 761-776.

28 Richeldi L. An update on the diagnosis of tuberculosis infection. Am J Respir Crit Care Med 2006; 174: 736-742.

29 Westermann J, Bode U. Distribution of activated T cells migrating through the body: a matter of life or death. Immunol Today 1999; 20: 302-306.

30 Williams MA, Bevan MJ. Effector and memory CTL differentiation. Annu Rev Immunol 2007; 25: 171-192.

31 Guyot-Revol V, Innes JA, Hackforth S, Hinks T, Lalvani A. Regulatory $\mathrm{T}$ cells are expanded in blood and disease sites in patients with tuberculosis. Am J Respir Crit Care Med 2006; 173: 803-810.

32 Sable SB, Goyal D, Verma I, Behera D, Khuller GK. Lung and blood mononuclear cell responses of tuberculosis patients to mycobacterial proteins. Eur Respir J 2007; 29: 337-346.

33 Schwander SK, Torres M, Carranza CC, et al. Pulmonary mononuclear cell responses to antigens of Mycobacterium tuberculosis in healthy household contacts of patients with active tuberculosis and healthy controls from the community. J Immunol 2000; 165: 1479-1485.

34 Schwander SK, Torres M, Sada E, et al. Enhanced responses to Mycobacterium tuberculosis antigens by human alveolar lymphocytes during active pulmonary tuberculosis. J Infect Dis 1998; 178: 1434-1445.

35 Ainslie GM, Solomon JA, Bateman ED. Lymphocyte and lymphocyte subset numbers in blood and in bronchoalveolar lavage and pleural fluid in various forms of human pulmonary tuberculosis at presentation and during recovery. Thorax 1992; 47: 513-518.

36 Barnes PF, Lu S, Abrams JS, Wang E, Yamamura M, Modlin RL. Cytokine production at the site of disease in human tuberculosis. Infect Immun 1993; 61: 3482-3489.

37 Gerosa F, Nisii C, Righetti S, et al. CD4 ${ }^{+} \mathrm{T}$ cell clones producing both interferon- $\gamma$ and interleukin-10 predominate in bronchoalveolar lavages of active pulmonary tuberculosis patients. Clin Immunol 1999; 92: 224-234.

38 Raju B, Tung CF, Cheng D, et al. In situ activation of helper T cells in the lung. Infect Immun 2001; 69: 4790-4798.

39 Rodrigues DS, Medeiros EA, Weckx LY, Bonnez W, Salomao R, Kallas EG. Immunophenotypic characterization of peripheral T lymphocytes in Mycobacterium tuberculosis infection and disease. Clin Exp Immunol 2002; 128: 149-154.

40 Urdaneta E, Feo-Figarella E, Montalvo C, et al. Characterization of local memory cells in stage-classified pulmonary tuberculosis: preliminary observations. Scand J Immunol 1998; 47: 496-501. 